\title{
Abscopal effect in lung cancer: three case reports and a concise review
}

\author{
Elena Garelli ${ }^{\ddagger}, 1$, Achim Rittmeyer ${ }^{*, \neq}, 2$ (D) Paul Martin Putora ${ }^{3,4}$, Markus Glatzer ${ }^{3}$, Ralf \\ Dressel $^{5}$ \& Stefan Andreas ${ }^{1,2}$ \\ ${ }^{1}$ Department of Pneumology \& Cardiology, University Medical Center Göttingen, Georg-August University, Göttingen, Germany \\ ${ }^{2}$ Department of Pneumology, Lungenfachklinik Immenhausen, Immenhausen, Germany \\ ${ }^{3}$ Department of Radiation Oncology, Kantonsspital St Gallen, St Gallen, Switzerland \\ ${ }^{4}$ Department of Radiation Oncology, University of Bern, Bern, Switzerland \\ ${ }^{5}$ Institute of Cellular \& Molecular Immunology, University Medical Center Göttingen, Göttingen, Germany \\ *Author for correspondence: arittmeyer@lungenfachklinik-immenhausen.de \\ $\ddagger$ Authors contributed equally
}

The abscopal effect describes the ability of locally administered radiotherapy to induce systemic antitumor effects. Over the past 40 years, reports on the abscopal effect following conventional radiation have been relatively rare, especially in less immunogenic tumors such as lung cancer. However, with the continued development and use of immunotherapy, reports on the abscopal effect have become increasingly frequent during the last decade. Here, we present three illustrative case reports from our own institution and previous published cases of the abscopal effect in patients with non-small cell lung cancer, treated with immune checkpoint inhibitors and radiotherapy. We also present a concise review of the clinical and experimental literature on the abscopal effect in non-small cell lung cancer.

First draft submitted: 26 June 2019; Accepted for publication: 7 November 2019; Published online: 21 November 2019

Keywords: abscopal effect $\bullet$ checkpoint inhibitors $\bullet$ immunotherapy $\bullet$ lung cancer $\bullet$ NSCLC $\bullet$ radiotherapy

The abscopal ( $a b$ - 'position away from' and scopus 'target') effect is a rare phenomenon in radiotherapy characterized by tumor regression outside the irradiated volume. It was first described by Mole in 1953 and has since been observed in different types of cancers after radiotherapy directed at the primary tumor or metastases [1]. A recent systematic review of 46 case reports of the abscopal effect following radiotherapy noted that the majority of cases occurred in immunogenic tumors such as renal cell carcinoma, melanoma, lymphoma and hepatocellular carcinoma [2]. Although the abscopal effect has been described in patients with non-small-cell lung cancer (NSCLC) treated only with radiotherapy $[3,4]$, this event seems to be rare due to the low immunogenicity of NSCLC and immunotolerance at the tumor site [5].

However, the effect is thought to occur with greater frequency when radiation is combined with immunostimulating agents such as immune checkpoint inhibitors [6,7]. With increased use of immunotherapy in addition to radiotherapy in lung cancer treatment, the abscopal effect is expected to become more common. In a recent systematic review, Chicas-Sett et al. investigated the efficacy and safety of stereotactic body radiation therapy (SBRT) and checkpoint inhibitor combination therapy in patients with lung cancer. They concluded that these two treatments combined present a good safety profile and achieve high rates of local control and greater chances of obtaining abscopal responses than radiotherapy alone $[8]$.

Since lung cancer has a high prevalence, and immunotherapy is used in an increasing proportion of patients and radiotherapy is often given for symptomatic and prognostic reasons, we first provide a concise overview of immunotherapy and radiotherapy in lung cancer. We then present three case reports from the Lungenfachklinik Immenhausen (Immenhausen, Germany) demonstrating the abscopal effect in patients with metastatic lung cancer treated with radiotherapy and immunotherapy. We also present the results of 11 previous clinical cases identified through a systematic literature search. Finally, we summarize the molecular effects of radiotherapy in the tumor microenvironment, the immunological mechanism of the abscopal effect, the rationale for combining radiotherapy

Future Medicine 
Table 1. Summary of three clinical cases showing abscopal effects observed at the Lungenfachklinik Immenhausen.

\begin{tabular}{|c|c|c|c|c|c|c|}
\hline Case & Lung cancer site & Previous treatment & $\begin{array}{l}\text { Site of } \\
\text { radiotherapy }\end{array}$ & $\begin{array}{l}\text { Site of abscopal } \\
\text { effect }\end{array}$ & $\begin{array}{l}\text { Radiotherapy } \\
\text { dose }\end{array}$ & Immunotherapy \\
\hline 1 & $\begin{array}{l}\text { LCNEC of the right upper lobe } \\
\text { with adrenal metastases } \\
\text { (cT4NOM1b) }\end{array}$ & $\begin{array}{l}\text { Pemetrexed, cisplatin and } \\
\text { bevacizumab; disease } \\
\text { progression after two cycles }\end{array}$ & $\begin{array}{l}\text { Second and third } \\
\text { thoracic vertebrae }\end{array}$ & Adrenal glands & $10 \times 3 \mathrm{~Gy}$ & Nivolumab \\
\hline 2 & $\begin{array}{l}\text { ADC of the left upper lobe with } \\
\text { brain and ocular metastases } \\
\text { (cT2N3M1C) }\end{array}$ & $\begin{array}{l}\text { Nab-paclitaxel/carboplatin }+ \\
\text { atezolizumab, then } \\
\text { atezolizumab; progression of } \\
\text { brain metastasis after } 5 \text { months } \\
\text { of treatment }\end{array}$ & Brain & $\begin{array}{l}\text { Lung and } \\
\text { mediastinal lymph } \\
\text { nodes }\end{array}$ & $10 \times 3$ Gy & Atezolizumab \\
\hline 3 & $\begin{array}{l}\text { ADC of the middle lobe with } \\
\text { malignant ipsilateral pleural } \\
\text { effusion (cT3N2M1a) }\end{array}$ & None & Brain & $\begin{array}{l}\text { Lung and pleural } \\
\text { disease }\end{array}$ & 10 × 3 Gy & Pembrolizumab \\
\hline
\end{tabular}

and immunotherapy, their applications in clinical trials exploring this important therapeutic option, and future challenges. To the best of our knowledge, this is the first comprehensive review focusing on the abscopal effect in patients with lung cancer.

\section{Immunotherapy \& radiotherapy in lung cancer} Immunotherapy in lung cancer

The use of immunotherapy in NSCLC is rapidly increasing. In particular, immune checkpoint inhibitors have shown some promise in modulating the tumor microenvironment, in order to fight against the tumor's attempt to evade the immune system [9].

Ipilimumab is a monoclonal antibody against cytotoxic T-lymphocyte-associated antigen 4 (CTLA-4) that blocks the CTLA-4 inhibitory pathway and restores effective T-cell function. Ipilimumab was approved by the US FDA in 2011 for the treatment of metastatic melanoma but has so far not been approved for the treatment of lung cancer.

Antibodies acting on programmed cell death protein 1 (PD-1) and its ligand, programmed death ligand 1 (PD-L1), have gained increasing attention in the treatment of lung cancer. Nivolumab and pembrolizumab are anti-PD-1 antibodies that bind and block PD-1 receptors on activated T-cells, while durvalumab and atezolizumab are anti-PD-L1 antibodies targeting PD-L1 on tumor cells. PD-1/PD-L1 pathway blockage allows $\mathrm{T}$ cells to destroy tumor cells [10]. To date, pembrolizumab, nivolumab and atezolizumab have received FDA approval for the treatment of metastatic NSCLC, with PD-L1 expression as a selection criterion for treatment with pembrolizumab.

Durvalumab has recently been approved for the treatment of unresectable stage III NSCLC patients whose disease has not progressed following platinum-based chemotherapy and radiation therapy [11].

\section{Radiotherapy in lung cancer}

Radiotherapy plays an important role in lung cancer treatment and it is used from early to advanced stages. Currently, resection is the standard of care for early-stage NSCLC; however, SBRT can be the preferred treatment for elderly or comorbid patients, resulting in local control rates of $90 \%$ at 5 years [12,13]. For locally advanced disease, especially with extensive lymph node metastases, chemo-radiotherapy (concurrent or sequential) followed by durvalumab has recently become the standard of care [14,15]. Radiotherapy is also used as a palliative treatment in stage IV lung cancer [16], for example, as brain radiotherapy or radiotherapy of bone metastases causing pain or at risk of instability. Radiotherapy has also demonstrated positive effects in oligometastatic disease [17]. These results are supported by two recent Phase II trials demonstrating that stereotactic ablative radiotherapy is associated with an improvement in overall survival in patients with oligometastatic cancers $[18,19]$.

\section{Case reports of abscopal effects in lung cancer}

We present recent case reports of three clinical cases of the abscopal effect in patients with metastatic lung cancer treated with radiotherapy and immunotherapy observed at the Lungenfachklinik Immenhausen (summarized in Table 1). 


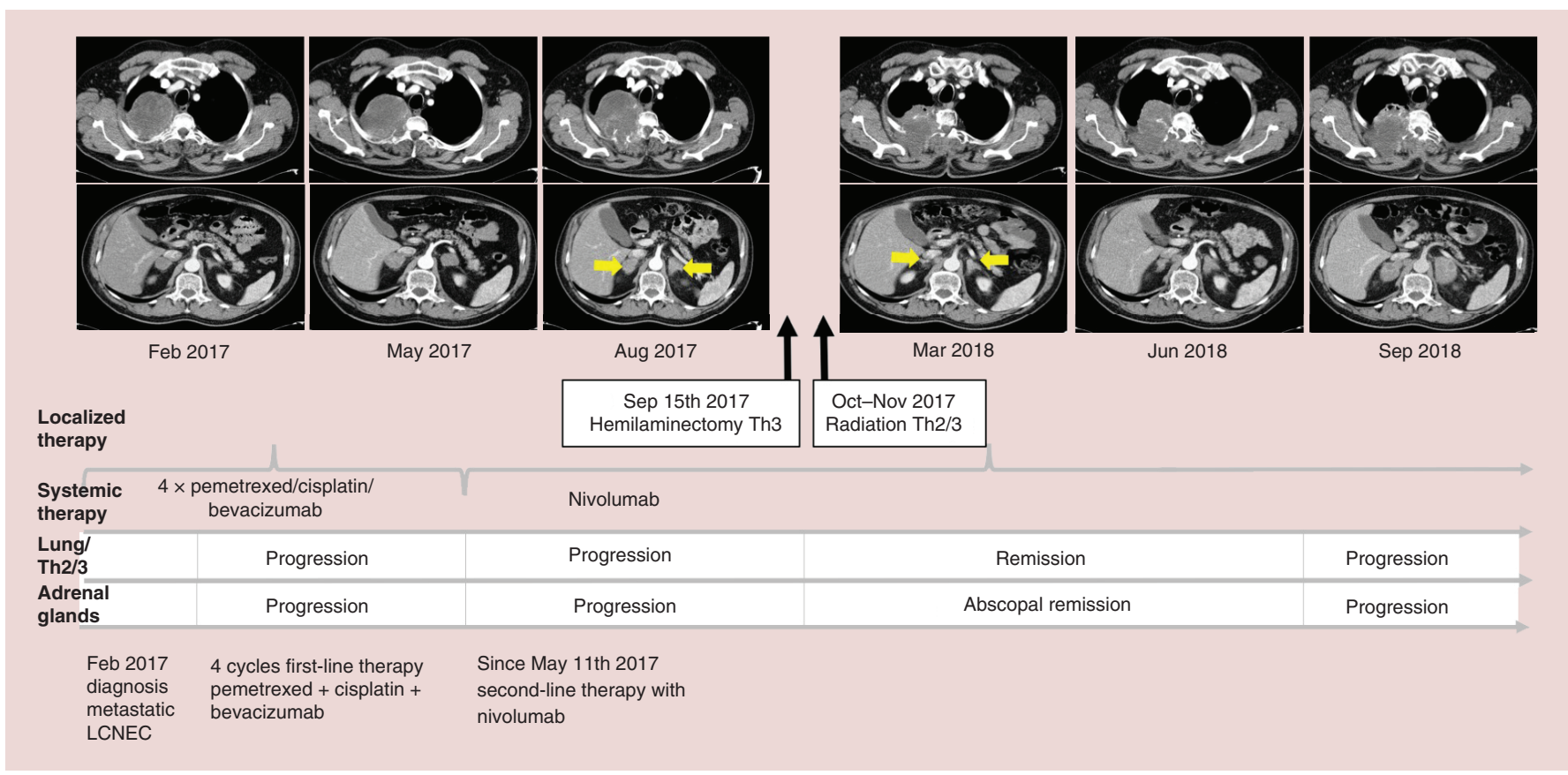

Figure 1. Case 1: thoracic and abdominal CT scans. Abscopal remission (arrows) of adrenal metastases after thoracic radiotherapy performed in October-November 2017.

LCNEC: Large-cell neuroendocrine carcinoma; Th: Thoracic vertebrae.

\section{Case 1}

A 54-year-old male patient presented with a pulmonary large cell neuroendocrine carcinoma of the right upper lobe, associated with bilateral adrenal metastases and a PD-L1 tumor proportion score of $20 \%$. The tumor, node, metastasis classification (8th edition [20]), based on positron emission tomography-CT and thoraco-abdominal CT, was cT4N0M1b (adrenal). After four cycles of chemotherapy (pemetrexed, cisplatin and bevacizumab), CT scans revealed disease progression in the right upper lobe as well as in both adrenal glands (Figure 1). Second-line therapy with nivolumab was started, leading to minor progression according to Response Evaluation Criteria in Solid Tumors 1.1 criteria, but with clinically progressive and increasingly symptomatic spinal cord compression due to tumor invasion.

At this point, pseudoprogression was discussed as well. In our clinical experience, after evaluation of more than 150 cases of patients that have been treated with nivolumab as second-line therapy for NSCLC, we have not found any patient with radiological pseudoprogression after 2 months. We routinely perform CT-scans after 7 and 14 weeks of therapy. Especially in this patient, we waited for a second CT-scan after 3 months to unequivocally rule out pseudo-progression before initiating radiotherapy.

Hemilaminectomy of the third thoracic vertebra combined with resection of the epidural tumor mass was performed leading to an R2 resection. Postoperative radiotherapy (30 Gy) was applied targeting the second and third thoracic vertebrae. Nivolumab was halted only for resection and reintroduced shortly before the first dose of radiation. CT scans 4 months after the first radiotherapy showed partial regression of the lung tumor and adrenal metastases. The patient showed disease progression 10 months after radiotherapy but is still alive 25 months after the initial diagnosis.

\section{Case 2}

A 64-year-old male patient presented with a central adenocarcinoma of the left upper lobe, considered cT2N3M1c due to pathological mediastinal contralateral lymph nodes and distant metastases (brain and ocular). The patient's PD-L1 results are currently blinded due to the requirements of a clinical trial (IMpower130 trial: ClinicalTrials. gov identifier NCT02367781). The radiological images after 5 months of treatment (four cycles of nab-paclitaxel/carboplatin with atezolizumab followed by four cycles of atezolizumab alone) showed an excellent response of the ocular metastasis, but progression of the brain metastasis (Figure 2). The thoracic tumor man- 


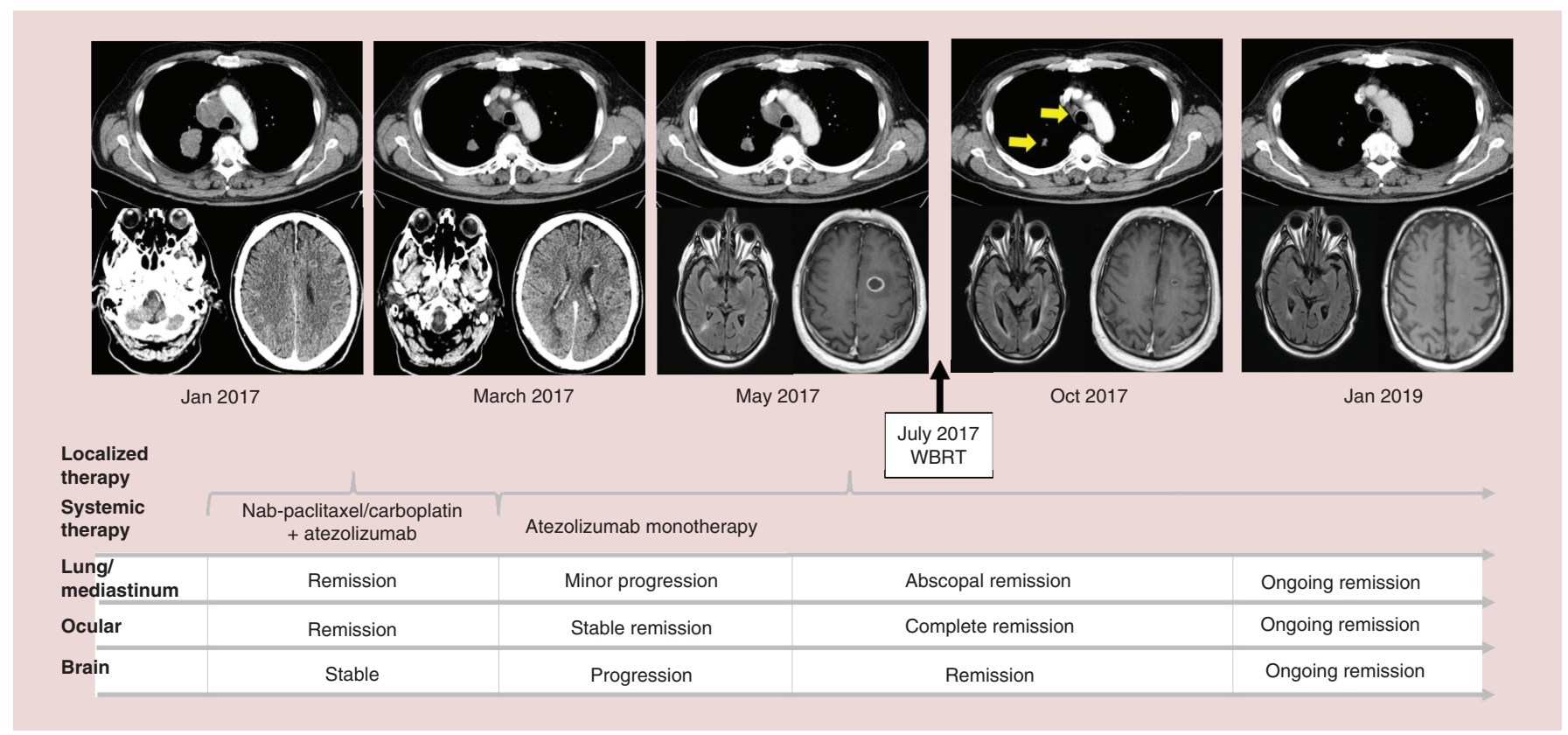

Figure 2. Case 2: CT scans. Abscopal remission (arrows) of thoracic disease after whole brain radiotherapy performed in July 2017.

ifestations showed partial remission after four cycles of combined chemotherapy and immunotherapy with no further shrinkage after four additional cycles of atezolizumab monotherapy. Whole-brain radiotherapy (WBRT) was performed and atezolizumab was continued. Radiological follow-up 4 months after WBRT showed a partial response in the brain as well as complete remission of lung and mediastinal tumor masses. The patient is still alive with radiologically nearly complete remission 28 months after the initial diagnosis of metastatic lung cancer.

\section{Case 3}

A 70-year-old male patient presented with a central adenocarcinoma of the middle lobe, associated with positive mediastinal lymph nodes and malignant ipsilateral pleural effusion (cT3N2M1a). There were no EGFR or $A L K$ mutations and the PD-L1 tumor proportion score was 70\%. First-line therapy with pembrolizumab was started, leading to a partial response. After a year of treatment, pulmonary and pleural disease progression occurred and a clinically symptomatic brain metastasis associated with perimetastatic cerebral edema appeared. Pembrolizumab was continued and WBRT added (30 Gy in ten fractions). Radiography of the thorax after radiotherapy showed partial regression of the lung tumor and pleural effusion. A thorax CT scan was performed 2 months after WBRT. The results in the 2-month follow-up CT scan are less evident when compared with the conventional chest $\mathrm{x}$-ray images 2 weeks after radiotherapy, demonstrating that the initial effect was short lived (Figure 3 ). The patient is still alive 19 months after the initial diagnosis.

\section{Previously published cases of abscopal effects in patients with lung cancer Search strategy}

We performed a systematic literature search for case reports and series of the abscopal effect following radiotherapy in patients with NSCLC. The inclusion criteria for the published articles were documentation of: primary NSCLC, site and dose of radiotherapy and site of the abscopal effect. Conference abstracts, editorials, reviews and cases not fulfilling of the inclusion criteria were to be excluded.

The search was performed in PubMed using the terms: 'abscopal AND lung', 'abscopal AND pulmonary', 'abscopal AND thoracic', 'nontargeted radiotherapy AND lung', 'nontargeted radiotherapy AND pulmonary', 'nontargeted radiotherapy AND thoracic', 'bystander AND lung', 'bystander AND pulmonary' and 'bystander AND thoracic'. No limit concerning year of publication and language was used. The final search was performed in May 2019.

In total, 1206 articles were identified from the PubMed search. After removal of 553 duplicates, the titles and abstracts of the remaining 653 articles were assessed for inclusion; 24 articles were eligible. Of these 24 articles, 13 


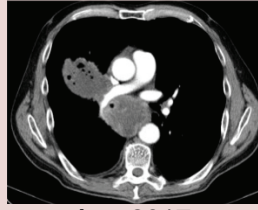

Aug 2017
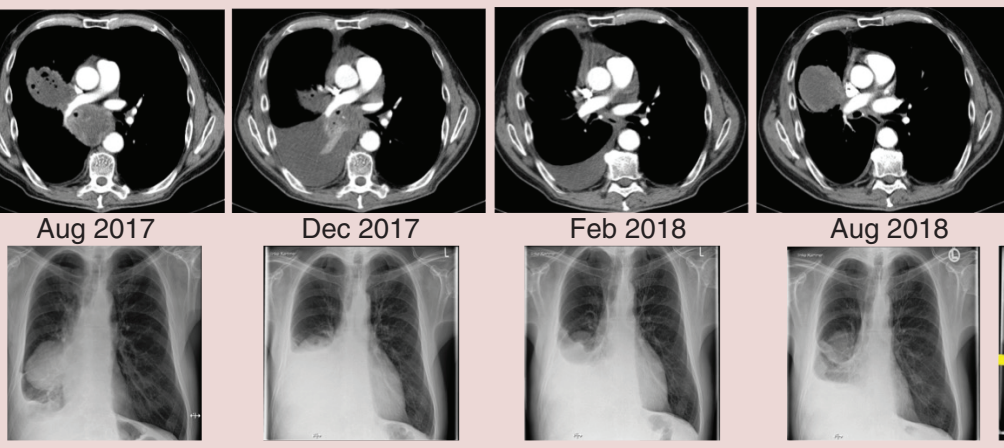

Feb 2018
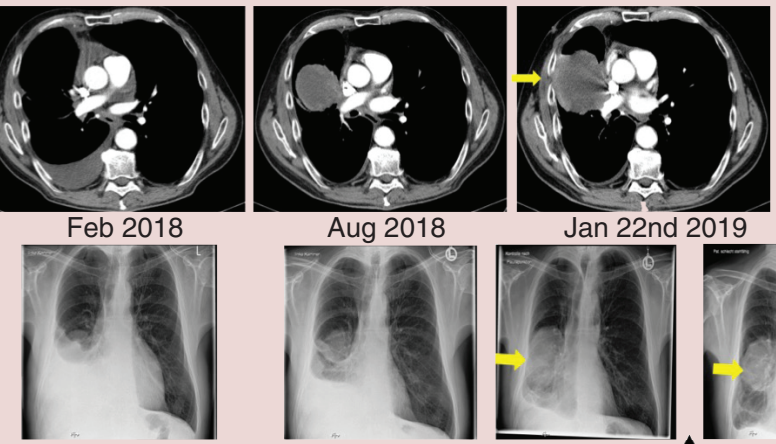

Jan 22nd 2019

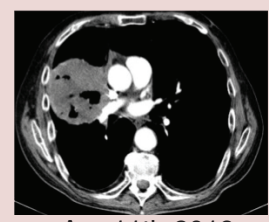

Apr 11th 2019

therapy

Systemic

therapy

Pembrolizumab monotherapy

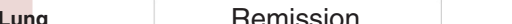

\begin{tabular}{l|l|l} 
Lymph node & Remission & Remission \\
position 7 & Remion
\end{tabular}

position 7

Pleural

effusion

Pseudoprogression

Remission

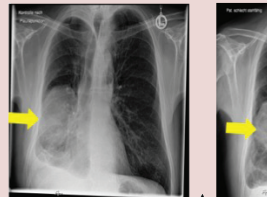

Jan 23rd 2019 Mar 6th 2019

Feb 11th to 25th 2019

WBRT

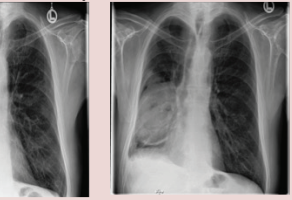

Apr 11th 2019

\begin{tabular}{|l|l|}
\hline Progression & Abscopal remission \\
\hline Remission & Ongoing remission \\
\hline Remission & Ongoing remission \\
\hline
\end{tabular}

Figure 3. Case 3: CT scans and x-ray images. Abscopal remission (arrows) of lung and pleural disease after whole brain radiotherapy performed in February 2019.

were further excluded: eight articles were reviews and did not include case reports, one article reported an abscopal effect but the primary tumor was melanoma, one case report described an abscopal effect in liver nodules considered to be hepatocellular carcinoma, two articles (including patients with NSCLC and small cell lung cancer) did not clearly demonstrate an abscopal effect, and one prospective cohort study described the abscopal effect in NSCLC but the site of response was not specified. Eleven articles fulfilled the primary search inclusion criteria (Figure 4).

\section{Review of previous clinical cases}

Table 2 presents a summary of the 11 case reports showing the abscopal effect in patients with lung cancer.

Rees and Ross reported the first case of a patient presenting with lung cancer and demonstrating an abscopal effect with radiotherapy in 1983 [3]. The patient, with advanced adenocarcinoma of the left lower lobe with multiple subcutaneous metastases, was treated with palliative radiation of the mediastinum and left lower lung using parallel opposed beams and a total dose of 35 Gy at 3.5 Gy per fraction. During treatment, a mass reduction was noticed in the subcutaneous metastases in the forehead and in the left shoulder which had not been treated by radiotherapy. Furthermore, at the 2 -week follow-up visit, the subcutaneous nodules were impalpable and a maculo-papular erythema appeared on both irradiated and nonirradiated skin. One week later, there was already evidence of regrowth of the left shoulder lesion and new subcutaneous metastases rapidly developed at other sites. The forehead lesion reappeared shortly before the patient's death 4 months after completion of radiation therapy [3].

Three studies showed an abscopal effect with radiotherapy alone in patients with NSCLC $[4,21,22,28]$. In 2013, Siva et al. described the abscopal effect in adrenal and bone metastases after SBRT of the lung [4]. In 2018, Hamilton et al. reported an abscopal effect in the lung after brain radiotherapy [21]. In 2019, Kuroda et al. reported the case of an abscopal response in the lung after mediastinum radiotherapy [22].

The first case of the abscopal effect of radiotherapy associated with immunotherapy was reported by Golden $e t a l$. in 2013, who combined radiotherapy with ipilimumab in a patient with metastatic adenocarcinoma of the lung [7]. The patient had progression after different lines of chemotherapy and previous lung radiation. Ipilimumab and local radiotherapy to one of the hepatic metastases was initiated, with the intent to generate an abscopal response. It is noteworthy that the authors report that this experimental approach was only based on two previous case reports in melanoma patients. Radiotherapy to a total dose of 30 Gy distributed in five fractions was delivered over a period of 10 days to the liver lesion, with one cycle of ipilimumab during radiation and three cycles afterward. Following 


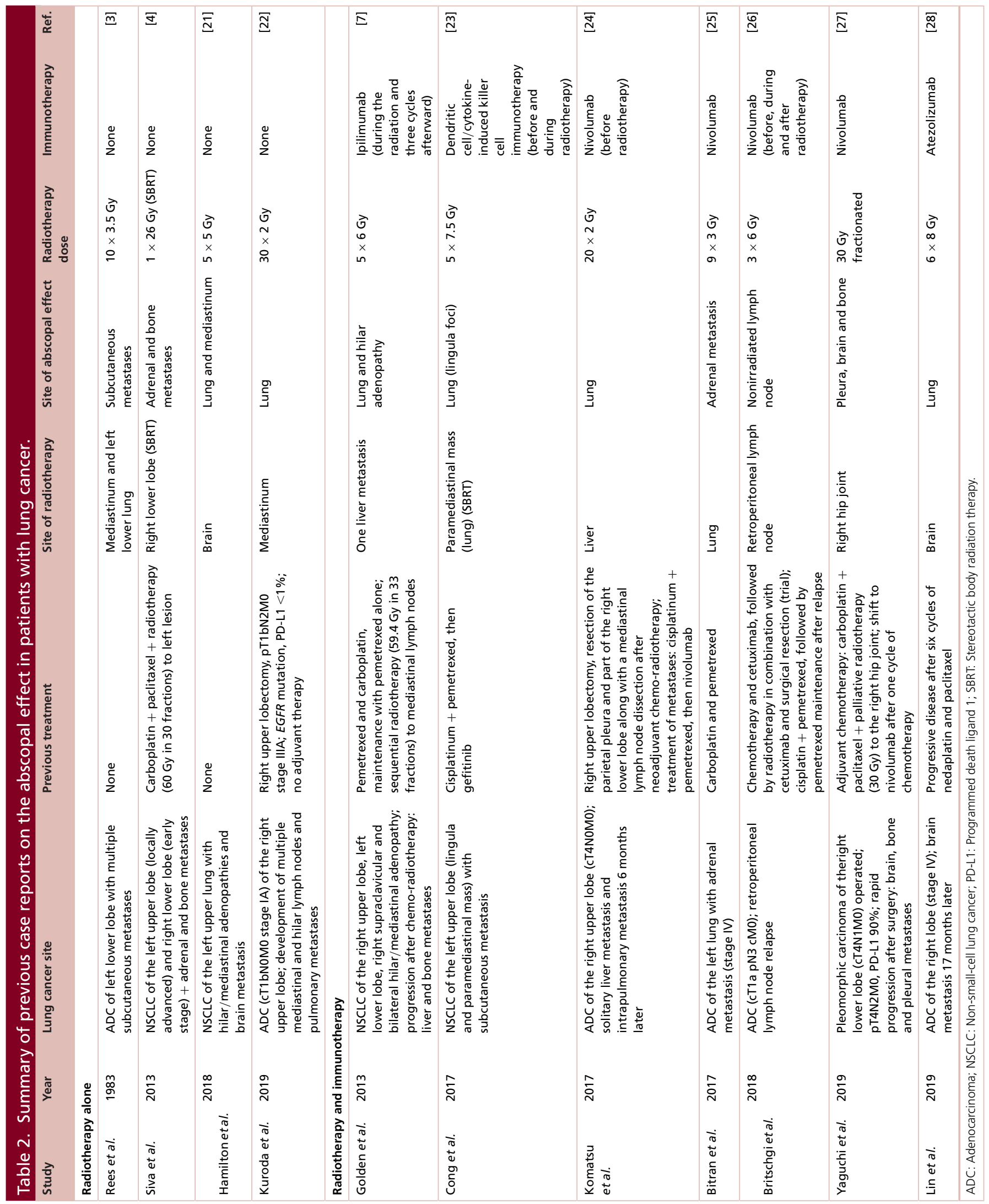




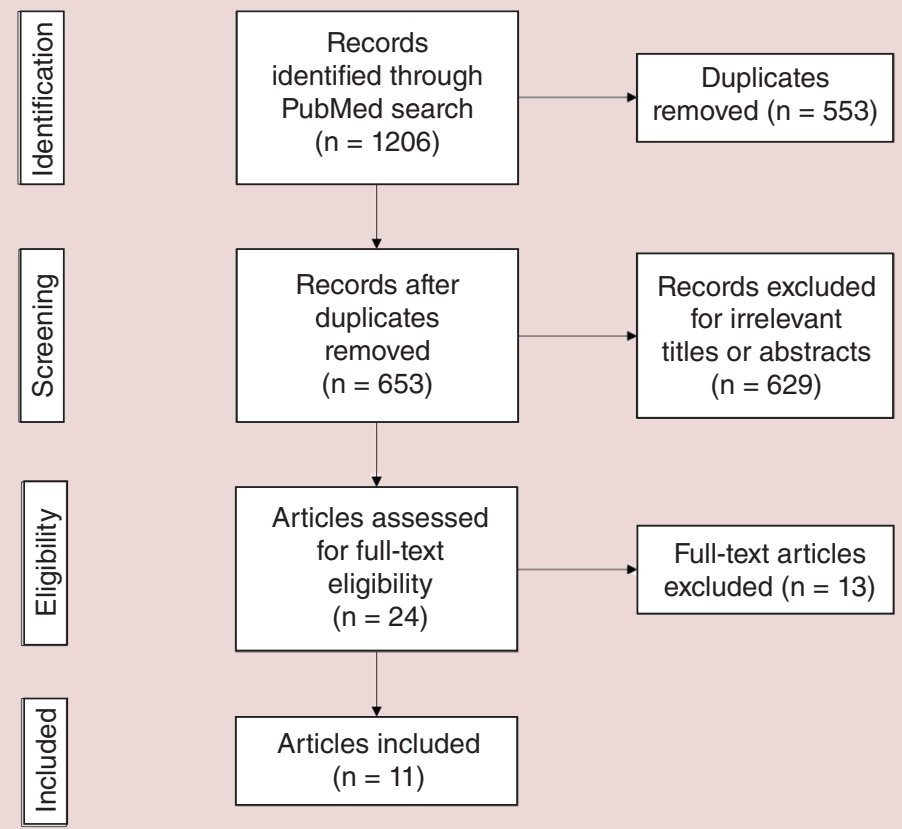

Figure 4. PRISMA flow diagram of the literature search strategy.

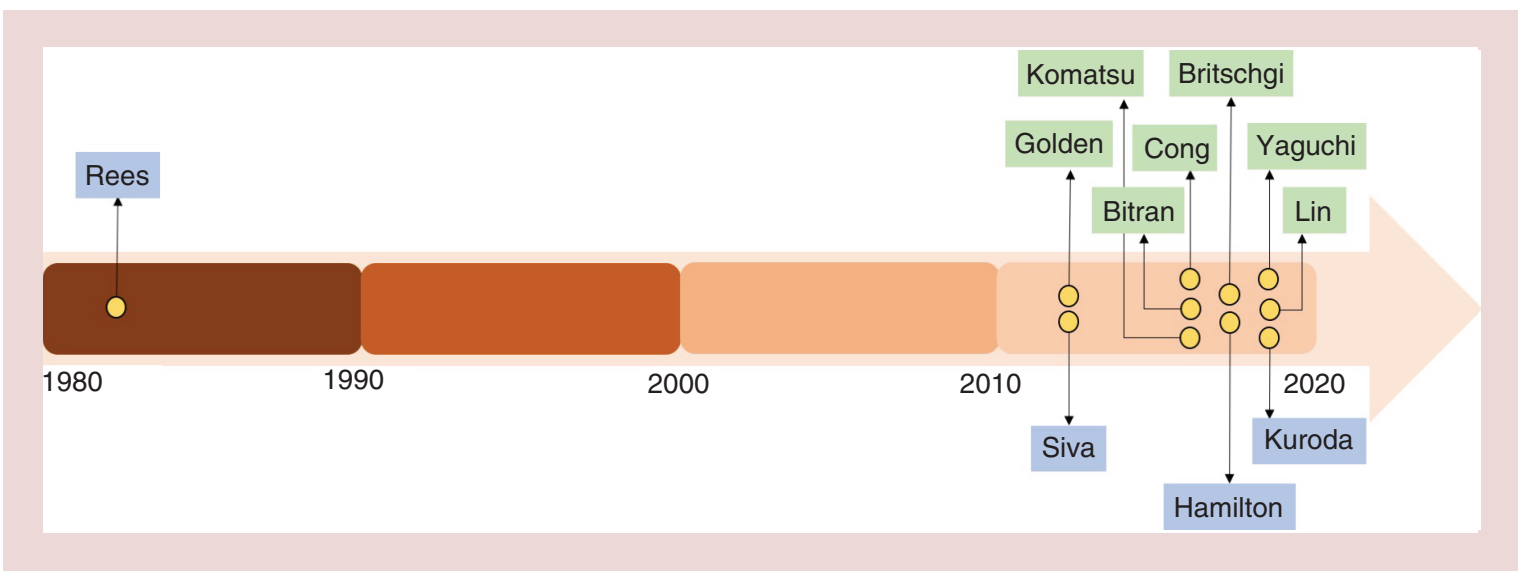

Figure 5. Timeline of publications of the abscopal effect in non-small-cell lung cancer during the last four decades (Table 2). The figure indicates a particular concentration within the last decade when radiotherapy is associated with immunotherapy (green cases) versus radiotherapy alone (blue cases).

this treatment, chest $\mathrm{CT}$ and positron emission tomography-CT showed a dramatic tumor response, not only in the radiotherapy field, but also at distant sites. The patient showed no evidence of disease at the 1-year follow-up [7].

More recent cases reported the abscopal effect in patients with lung cancer treated with radiotherapy and immunotherapy, in particular with nivolumab [23-28]. These cases are very heterogeneous in terms of the site of radiotherapy and abscopal effect, with abscopal responses described in the lung, brain or adrenal gland after bone, liver or lung radiotherapy.

Reports about the abscopal effect in lung cancer have appeared regularly since 2013, next to a potential awareness among clinicians that the introduction of immunotherapy seems to play a role in this phenomenon (Figure 5). 


\section{Radiation \& the immune system}

The main focus of radiotherapy is the control and eradication of local tumor tissue by maximizing direct tumor cell damage and minimizing healthy tissue impairment [29]. The main target of ionizing radiation is the DNA itself: radiation induces base damage, single-strand breaks or double-strand breaks that may lead to cell death, especially in the setting of defective repair mechanisms [30]. The toxic effect of radiation can equally affect surrounding leukocytes, resulting in subsequent immunosuppression. Lymphopenia following radiotherapy for solid tumors such as lung or breast cancer has been described [31,32]. This effect is mainly explained by the high radiation sensitivity of lymphocytes (less than $1 \mathrm{~Gy}$ ). It is noteworthy that the entire circulating blood pool receives a potentially lymphotoxic dose during certain radiotherapy courses, for example, large volume thoracic radiotherapy [33].

More recently, radiotherapy has also been shown to induce a number of systemic immune-modulatory effects in the tumor. Radiotherapy can contribute to creating an immunosuppressive environment by making $\mathrm{T}$ cells dysfunctional and by recruiting immunosuppressive myeloid cells that directly promote tumor growth [34,35]. Radiotherapy might also induce PD-L1 expression in the tumor microenvironment, allowing engagement of inhibitory PD-1 receptors on T-cells and inhibiting the T-cell antitumor response [36]. However, radiation may generate positive immune-modulatory effects leading to beneficial tumor-specific immune responses [37]. The abscopal effect is believed to arise from the local capacity of radiotherapy to stimulate this systemic immune response to control unirradiated cancer. Demaria $e t$ al. were the first to identify an immune-mediated mechanism as the basis of this effect and more recent studies have demonstrated how radiotherapy can activate a tumor-specific systemic immune response [38-40]. Radiotherapy acts as an immune modulator in the tumor microenvironment through several mechanisms that are summarized in Figure 6.

In particular, upregulation of the endoplasmic reticulum chaperone calreticulin and its translocation to the plasma membrane of dying irradiated tumor cells facilitates phagocytosis of material from tumor cells via binding to CD91 [41]. Heat shock proteins released from dying cells, such as HSP70, can bind to several receptors on dendritic cells, including CD91 and Toll-like receptor 4 (TLR4), and provide danger signals that may not only activate antitumor immune responses by $\mathrm{CD}^{+} \mathrm{T}$ cells but also by natural killer (NK) cells $[42,43]$.

High-mobility group box 1 protein, released from dying tumor cells, binds to Toll-like receptor 4 , leading to dendritic cell maturation $[44,45]$. This factor also stimulates monocytes to produce cytokines such as TNF- $\alpha$ and the interleukins IL-1, IL-6 and IL-8, acting as pro-inflammatory mediators, which also contribute to dendritic cell maturation [46]. Moreover, dying tumor cells release ATP, which binds to purinergic receptors, including P2RY2 and P2RX7, and promotes the infiltration of tumors with dendritic cells and subsequent inflammasome activation and IL-1 $\beta$ secretion [47]. Radiotherapy also stimulates tumor cells to release the chemokines CXCL16 and CXCL10, and increases the expression of adhesion molecules such as E-selectin, intercellular adhesion molecule 1 and vascular cell adhesion protein 1 on endothelial cells. Surviving irradiated cells increase the expression of death receptors, including Fas, and upregulate major histocompatibility complex (MHC) class I molecules, improving their recognition and killing by activated tumor antigen-specific $\mathrm{CD}^{+} \mathrm{T}$ cells [48-54]. Upregulation of ligands of the activating NK receptor NKG2D on irradiated tumor cells enables them to deliver their co-stimulatory signals to $\mathrm{CD}^{+} \mathrm{T}$ cells [55] and renders them susceptible to NK cells [56]. Activated dendritic cells migrate to local lymph nodes where they cross-present tumor antigens on MHC class I molecules to naive $\mathrm{CD}^{+} \mathrm{T}$ cells. Those with specificity for tumor antigens proliferate and differentiate into cytotoxic effector T-cells. These effector T-cells migrate back to the tumor, attracted by chemokines released due to irradiation-induced cell destruction. In addition, these effector $T$ cells can induce tumor cell death in nonirradiated lesions distal to the initial irradiation site $[51,52]$. The infiltration of tumors by effector $T$ cells seems to be promoted by radiotherapy-induced remodeling of the vascularization in the tumor microenvironment $[57,58]$. However, tumors still can escape an immune response mediated by activated cytotoxic $\mathrm{T}$ cells by mechanisms that, in addition to the loss of tumor antigens or MHC class I expression, also include the upregulation of PD-1 ligands, which can engage the inhibitory PD-1 receptor on the effector T-cells and lead to T-cell exhaustion [59].

The dose per fraction of radiotherapy seems to have an important impact on systemic consequences. Radiationinduced nuclear DNA release and subsequent sensing of cytoplasmic double-stranded DNA can activate the cyclic guanosine monophosphate-adenosine monophosphate synthase (cGAS). This DNA sensor induces production of the second messenger cyclic guanosine monophosphate-adenosine monophosphate (cGAMP), which binds and activates the STING (stimulator of interferon genes) pathway, producing type I IFN, a key mediator of dendritic cell recruitment and maturation [60-62]. Notably, the study of Vanpouille-Box et al. demonstrated that a radiation 


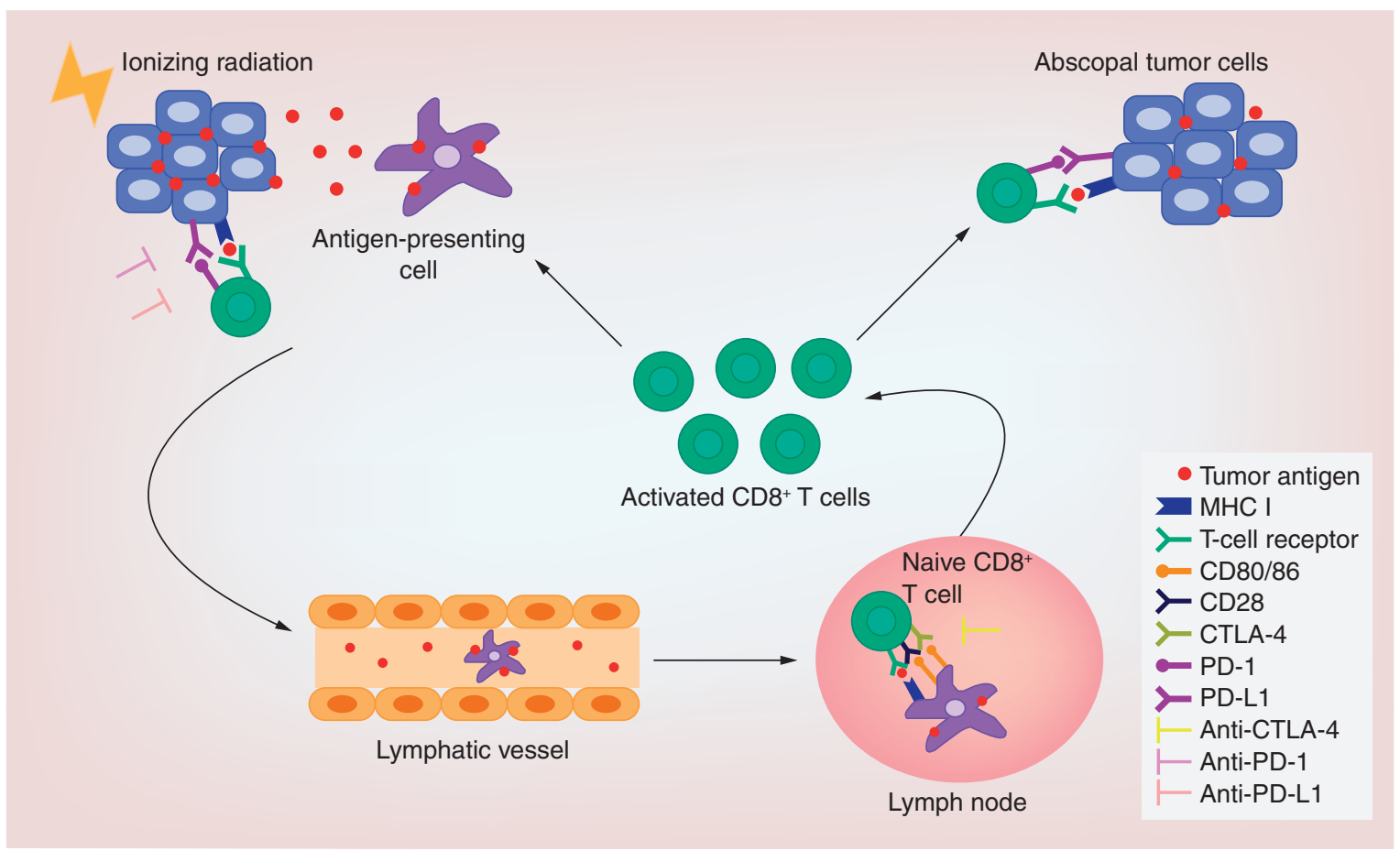

Figure 6. Mechanism of the abscopal effect and its relationship with immune checkpoint inhibitors. Radiotherapy can lead to immunogenic cell death and the release of tumor antigens by irradiated tumor cells. These antigens are taken up by professional antigen-presenting cells such as dendritic cells. Upon maturation, the dendritic cells migrate to the lymph nodes where they present the processed antigens in the context of major histocompatibility complex molecules to $\mathrm{T}$ cells. Activation of $\mathrm{CD}^{+} \mathrm{T}$ cells requires the cross-presentation of exogenous tumor antigens on major histocompatibility complex class I molecules. Naive $\mathrm{CD}^{+} \mathrm{T}$ cells receive the antigen-specific signal through the T-cell receptor and co-stimulatory signals such as CD80 and CD86 through CD28. Tumor antigen-specific CD8 ${ }^{+} \mathrm{T}_{\text {cells }}$ proliferate and differentiate into cytotoxic effector T cells that migrate from the lymph nodes to the tumor sites (primary tumor and nonirradiated tumor metastases) in order to exercise their effect of killing tumor cells. However, cytotoxic T-lymphocyte-associated antigen 4 binds to CD28 competitively with CD80/86 and inhibits the activation of T cells. Following T-cell activation, programmed cell death protein 1 receptors are expressed on the T-cell surface, which bind primarily to programmed death ligand 1 and inhibit the activity of the $\mathrm{CD} 8^{+} \mathrm{T}$ cells. Therefore, the administration of immune checkpoint inhibitors (anti-cytotoxic T-lymphocyte-associated antigen 4, antiprogrammed cell death protein 1 and anti-programmed death ligand 1) can enhance the antitumor immune response of radiotherapy.

dose above a threshold of 10-12 Gy per fraction could attenuate the immunogenicity of cancer cells because of the induced upregulation of the DNA exonuclease three-prime repair exonuclease 1. Three-prime repair exonuclease 1 degrades cytosolic double-stranded DNA, thereby removing the trigger for the cGAS-STING pathway, abrogating the immunogenicity of irradiated cancer cells [63].

Although the abscopal effect of radiotherapy alone has been reported in a growing number of cases [2], the overall occurrence rate is relatively low. This may be explained by the counterbalance of the pro-immunogenic effects produced by ionizing radiation with the immunosuppressive consequences of radiotherapy.

\section{Timing of immunotherapy in combination with radiotherapy}

\section{Theoretical principles}

The combination of radiotherapy and checkpoint blockade can act at different stages of the antitumor immune response. The release of new tumor antigens by radiotherapy may interact with anti-CTLA- 4 to increase the diversity of tumor antigen-specific naive $\mathrm{T}$ cells that become activated. At the same time, memory $\mathrm{T}$ cells with specificity for tumor antigens might become reactivated. In addition, anti-CTLA-4 can inhibit the development of regulatory $\mathrm{T}$ cells (Tregs), which promote tolerance against tumor antigens. Radiotherapy can increase the infiltration of the activated or reactivated T-cells into the tumor. Anti-PD-1 and anti-PD-L1 help to prevent the 
suppression of effector T cells induced by upregulation of PD-L1 in tumors mediated by radiotherapy. Moreover, anti-PD-1/PD-L1 can reactivate exhausted intratumoral T cells $[64,65]$.

\section{Preclinical evidence}

Due to the different timing of these mechanisms in the immune response, it might be important to determine the most effective sequence of radiotherapy and checkpoint inhibition. Theoretically, it would be expected that radiotherapy and anti-CTLA-4 should follow in quite short order and that anti-PD-1/PD-L1 would be effective at later time points. These concepts are largely supported by results of different pre-clinical models. In murine models of breast cancer, concurrent and postradiotherapy treatment with anti-CTLA- 4 has been shown to control tumor growth, compared with monotherapy $[6,66]$. Other studies have reported an enhanced antitumor response from combining radiotherapy with anti-CTLA-4, showing that this effect was principally due to a reduction of Tregs and an increase of cytotoxic T-cell populations in both primary and secondary tumors [67,68]. Notably, treatment with anti-CTLA-4 can be effective even before radiotherapy due to the depletion of Tregs [69].

Other studies have investigated the optimal timing of radiotherapy and anti-PD-1/PD-L1 combination. In two different preclinical studies, Dovedi et al. showed how the administration of anti-PD-L1 at the same time point as radiotherapy (i.e., at the beginning and at the end of radiotherapy) was superior to sequential treatment, which might suggest that an upregulation of anti-PD-L1 occurs rapidly after radiotherapy. Interestingly, the mechanism responsible of PD-L1 upregulation on tumor cells was reported to involve the production of IFN- $\gamma$ by tumorinfiltrating $\mathrm{CD}^{+} \mathrm{T}$ cells. This acquired resistance to radiotherapy could be avoided by concurrent administration of anti-PD-L1 [70,71]. To define the best timing of anti-PD-1/L1 administration, a close look at the kinetics of T-cell tumor infiltration following radiotherapy might be required. Hettich $e$ al. reported that after radiotherapy alone with 12 Gy over two consecutive days, the overall tumor-infiltrating lymphocyte (TIL) peak appeared 5 days after radiotherapy and then gradually decrease to preradiotherapy levels. Almost all the TILs were $\mathrm{CD} 8^{+} \mathrm{T}$ cells, while the number of $\mathrm{CD}^{+}{ }^{+}$Tregs was low. Considering that PD-1 was strongly expressed on TILs from irradiated tumors already on day 5 postirradiation, the authors administered anti-PD-1 on the day of the second radiotherapy fraction. The radiotherapy/anti-PD-1 combination was highly synergistic, inducing substantial tumor regression in five out of nine mice [72]. Recently, the combination of radiotherapy and anti-CTLA-4 or PD-1 blockade has been evaluated in mouse models. Radiotherapy mainly enlarged the intratumoral T-cell receptor repertoire, likely by increasing the amount and diversity of tumor antigens released from tumors and presented by dendritic cells. Anti-CTLA-4 in combination with radiotherapy promoted the expansion of tumor antigen-specific T-cell clones, while blockade of the PD-1/PD-L1 pathway was required to prevent exhaustion of activated effector T cells [6]. Taken together, these studies suggest that, even if the precise T-cell tumor kinetics depend on the model and radiotherapy dose, anti-PD-1 should be administered concurrently or shortly following radiotherapy to take advantage of the peak in tumor effector $\mathrm{CD} 8^{+} \mathrm{T}$ cells [73].

\section{Clinical evidence}

Clinical case reports have found that the administration of anti-CTLA- 4 before radiotherapy correlated with improved survival in patients with metastatic melanoma [74,75].

Two Phase I trials have evaluated the delivery of ipilimumab after and before SBRT in patients with metastatic melanoma [73,76]. Interestingly, ipilimumab administered after SBRT resulted in only an $18 \%$ partial response rate, whereas administration of ipilimumab before radiotherapy showed a $50 \%$ complete or partial response rate and a $27 \%$ complete response rate [76]. At present, this treatment has not yet found its way into clinical practice. Currently, different trials investigating the efficacy of radiotherapy in combination with immunotherapy are being finalized. However, these studies seem to only marginally evaluate the optimal timing of radiotherapy. In our case reports (Table 1), anti-PD-1 antibodies were administered before and continued during and after radiotherapy. Thus, at present it seems difficult to determine the best treatment schedule for radiotherapy and immunotherapy. Moreover, the timing of the antitumor immune responses that are ongoing is likely to be much more complex in patients than in animal models so that it is difficult to apply directly preclinical evidence obtained in mouse models. In patients, one may observe different phases of the immune response for different $\mathrm{T}$ cell clones at the same time point.

\section{Interaction of nodal irradiation \& the immune system}

It is unclear which type of radiotherapy regimen should be used when radiotherapy is combined with immunotherapy. It is also unknown whether elective nodal irradiation affects adaptive immune responses and the combinatorial 
efficacy of these two treatments. As shown in Figure 6, tumor-draining lymph nodes (TDLNs) are the main location at which antigen-presenting cells prime $T$ cells. The importance of TDLNs in the generation of a specific antitumor immune response has been demonstrated by preclinical studies. Takeshima et al. showed that TDLN-deficient mice and mice with surgically ablated TDLNs presented with reduced tumor control following radiotherapy [77]. In a preclinical model, Marciscano et al. demonstrated that elective nodal irradiation decreased the immune response and affected the combinatorial efficacy of SBRT with immunotherapy. They showed that radiotherapy-mediated chemokine expression was attenuated by the irradiation of both the tumor and TDLNs, resulting in impaired trafficking of $\mathrm{CD}^{+} \mathrm{T}$ cells into the tumor and reducing survival [78]. Clinical data has demonstrated that for most cases elective nodal irradiation is not required and recent guidelines do not recommend this routinely [79], whether this might affect potential abscopal effects in the future remains unclear.

\section{Optimal dose \& fractionation}

Radiotherapy is traditionally delivered with 1.8-2.2 Gy per fraction (normofractionation); however, fewer fractions of higher doses (hypofractionation) are common in the palliative or stereotactic setting, while hyperfractionation is established in the primary treatment of small cell lung cancer [80]. The dose and fractionation seem to impact the immunotherapeutic potential and the presence of an abscopal effect [81]. Considering that lymphocytes are exquisitely sensitive to radiation, repetitive daily radiotherapy can deplete migrating immune effector cells. Frey et al. showed that following $2 \times 5$ Gy fractions, the intratumoral CD8 ${ }^{+}$T-cell peak appeared at day 8 and declined by day 9 , and an increase of Tregs was seen following the infiltration of CD8 ${ }^{+} \mathrm{T}$ cells, at days $8-10$. The optimal timing to re-irradiate the tumor in this case would be at days 9-10 when the cytotoxic T-cells have already migrated away and immunosuppressive Tregs are still inside the tumor [82]. Siva et al. demonstrated that single-dose (12 Gy) radiotherapy did not deplete established immune effector cells $\left(\mathrm{CD}^{+}, \mathrm{CD} 4^{+}\right.$and $\mathrm{NK}$ cells) critical to the curative activity of radiotherapy when associated with immunotherapy [83]. Indeed, compared with conventional modalities, radiotherapy with ablative high dose per fractionation seems to be more effective in enhancing the antitumor immune response [84]. Other studies have investigated the combination of hypofractionated radiotherapy and immunotherapy. Dewan et al., for example, demonstrated in murine breast and colon cancer models that $5 \times 6 \mathrm{~Gy}$ and $3 \times 8$ Gy protocols of radiotherapy were more effective in inducing the immune-mediated abscopal effect than a single ablative dose when combined with immunotherapy [66]. Similarly, the study of Schaue et al., on a murine melanoma model, found that fractionated treatment with medium radiation doses of 7.5 Gy per fraction induced the best tumor control and antitumor response [81].

The dose per fraction of radiotherapy seems to be correlated with the generation of immunological effects. As previously reported, a radiation dose above a threshold of 10-12 Gy per fraction could abrogate the immunogenicity of irradiated cancer cells, removing the trigger for the cGAS-STING pathway [63]. These considerations have important clinical implications for dose selection and fraction when radiotherapy is associated with immunotherapy.

Optimal radiation dose, timing and fractioning remain points of debate and clear recommendations are still lacking [85].

\section{Clinical trials combining radiotherapy \& immune checkpoint inhibitors}

Various clinical trials have investigated the efficacy and safety of combining radiotherapy and checkpoint inhibitors in patients with NSCLC. This combination may be accompanied by important side effects such as pneumonitis [86,87] and, potentially, myocarditis [88]. However, several retrospective studies showed no increased toxicities for the combination of immunotherapy and radiotherapy in the treatment of stage IV lung cancer patients [89].

In a prospective study, Formenti et al. tested the combination of radiotherapy with concurrent ipilimumab in 39 patients with metastatic NSCLC and progression after systemic treatment. Only 21 patients were evaluable as 18 patients discontinued ipilimumab treatment, mostly due to the adverse effects of ipilimumab. Of the 21 evaluable patients, ten presented an abscopal response based on Response Evaluation Criteria in Solid Tumors 1.1 criteria, using the best responding lesion [90].

In an unplanned secondary analysis of the Phase I KEYNOTE-001 trial, a subgroup of 24 of 97 patients with metastatic NSCLC receiving thoracic radiotherapy followed by pembrolizumab were analyzed. Pembrolizumab was associated with significantly longer progression-free survival (PFS) and overall survival in patients who previously received extracranial radiotherapy compared with those who did not receive extracranial radiotherapy (PFS 6.3 vs 2.0 months; $\mathrm{p}=0.008$; overall survival 11.6 vs 5.3 months; $\mathrm{p}=0.034)$. Pulmonary toxicity of any grade was high and of borderline significance $(\mathrm{p}=0.052)$ in patients with previous thoracic radiotherapy $(63 \%, 5 / 24)$ versus 
patients with no previous thoracic radiotherapy (40\%, 29/73). The incidence of pembrolizumab-related pulmonary toxicity of any grade was significantly higher in patients with previous thoracic radiotherapy $(13 \%, 3 / 24)$ versus patients with no previous thoracic radiotherapy $(1 \%, 1 / 73 ; \mathrm{p}=0.046)$ [91]. Considering that the overall response rate of single-agent PD-1/PD-L1 blockade is only 17-19\%, these data support the use of radiotherapy as a strategy to induce a response to immune checkpoint inhibitors in patients with metastatic lung cancer $[92,93]$.

This strategy is further supported by the results of a randomized Phase II trial of SBRT and sequential pembrolizumab versus pembrolizumab alone for patients with advanced NSCLC. The group treated with radiotherapy and pembrolizumab showed an overall response rate of $41 \%$ and a median PFS of 6.4 months compared with 19\% and 1.8 months, respectively, for the control arm [94].

The PACIFIC trial is a Phase III randomized study comparing durvalumab as consolidation therapy with placebo in patients with stage III, locally advanced, unresectable NSCLC that had not progressed after platinumbased chemo-radiotherapy. Immunotherapy increased the 2-year overall survival significantly from 55.6 to $66.3 \%$. Durvalumab also had a favorable effect on the frequency of new metastases, including a lower incidence of new brain metastases, and the median time to death or distant metastases was longer in patients treated with durvalumab (23.2 vs 14.6 months, hazard ratio: 0.52 ; $95 \%$ CI $0.39-0.69 ; \mathrm{p}<0.001$ ). With regard to safety, the incidence of adverse events of any cause was similar in the two groups: $96.8 \%$ in patients who received durvalumab and $94.9 \%$ in patients who received placebo; grade 3 or 4 adverse events occurred in 29.9 and $26.1 \%$, respectively, and the most frequent were pneumonia ( $4 \%$ in each arm) and pneumonitis (3\% in each arm) [95].

To date, about 40 planned or ongoing trials investigating radiotherapy combined with immune checkpoint inhibitors in patients with NSCLC are registered at the ClinicalTrials. gov registry.

The recent systematic review of Chicas-Sett $e t$ al., investigating the combination of radiotherapy and checkpoint inhibitors in NSCLC, reported a mean PFS of 4.6 months and a weighted mean overall survival of 12.4 months in combination therapy. When compared with patients with NSCLC and checkpoint inhibitor therapy only, PFS was slightly higher in the combination group and overall survival was reported to be similar, especially considering prospective studies. This questions an additional survival benefit of radiotherapy and immunotherapy together, even if the abscopal effect is more prevalent in this group. Grade $\geq 3$ toxicity ranged between 10 and $17 \%$ for anti-PD-1/PD-L1 and 29 and 38\% for anti-CTLA-4, which is consistent with the adverse events that can be expected from checkpoint inhibitors alone [8]. These results have to be considered carefully as retrospective and prospective studies and studies with different cancer types were included in this review.

\section{Conclusion}

The abscopal effect of radiotherapy has been reported in preclinical and clinical studies in different tumor entities. The mechanism responsible for this effect is immune mediated: irradiated tumor cells can, in fact, stimulate antitumor adaptive immunity by inducing the release of tumor antigens and their cross-presentation to $\mathrm{CD} 8^{+} \mathrm{T}$ cells. Only a few cases of the abscopal effect after radiotherapy alone have been reported in patients with lung cancer so far. However, with the development of immunotherapy, especially of immune checkpoints inhibitors, the abscopal effect of radiotherapy might become more frequent. Immunotherapy can potentiate the in situ vaccination effect of radiotherapy.

\section{Future perspective}

Many challenges remain for this combined treatment as the optimal dose/fractionation schemes for radiotherapy, the optimal time of administration of immunotherapy and the prediction of treatment efficacy are still unclear. These questions need to be addressed in future clinical studies with the aim of developing evidence-based guidelines for radiotherapy and immunotherapy.

\section{Author contributions}

A Rittmeyer and S Andreas conceived of the study. E Garelli, A Rittmeyer, PM Putora, M Glatzer and R Dressel contributed to the text and figures. E Garelli, A Rittmeyer, R Dressel and S Andreas drafted the manuscript, and all authors revised the manuscript.

\section{Acknowledgments}

The authors sincerely thank the patients and their families, the staff of the LKI Immenhausen (Immenhausen, Germany), and Eugen Bloch (Clinic for Radiotherapy, Kassel, Germany) who kindly provided radiotherapy planning figures for case presentation 4. 
Financial \& competing interests disclosure

The authors have no relevant affiliations or financial involvement with any organization or entity with a financial interest in or financial conflict with the subject matter or materials discussed in the manuscript. This include employment, consultancies, honoraria stock ownership or options, expert testimony, grants or patents received or pending, or royalties.

The authors would like to thank Ray Loughlin (Academic Editor, Sheffield, UK) for medical writing assistance.

\section{Ethical conduct of research}

The authors state that they have obtained appropriate institutional review board approval or have followed the principles outlined in the Declaration of Helsinki for all human or animal experimental investigations. In addition, for investigations involving human subjects, informed consent has been obtained from the participants involved.

\section{Informed consent}

All patients provided written informed consent for publication for radiologic and clinical data.

\section{Open access}

This work is licensed under the Creative Commons Attribution-NonCommercial 4.0 Unported License. To view a copy of this license, visit http://creativecommons.org/licenses/by-nc-nd/3.0/

\section{Executive summary}

Immunotherapy \& radiotherapy in lung cancer

- Immunotherapy, especially using checkpoint inhibitors, has shown some promise in modulating the tumor microenvironment, in order to fight against the tumor's attempt to evade the immune system.

- Radiotherapy plays an important role in lung cancer treatment and is used from early to advanced stages.

Abscopal effect

- The abscopal effect is a rare phenomenon in radiotherapy characterized by tumor regression outside the irradiated volume.

- The abscopal effect of radiotherapy could be enhanced by combining immunotherapy and radiotherapy.

- The incidence of the abscopal effect has increased over the last decade after the introduction of checkpoint inhibitors in lung cancer treatment.

Radiation \& the immune system

- The mechanism underlying the abscopal effect is immune mediated. Radiotherapy can lead to immunogenic cell death and the release of tumor antigens by irradiated tumor cells, stimulating an antitumor immune response. The administration of immune checkpoint inhibitors acting at different points enhances this response.

Timing of immunotherapy in combination with radiotherapy

- Various preclinical and clinical studies have investigated the optimal strategies for combining radiotherapy and immunotherapy, but timing, dose and fractionation remain unclear.

\section{Clinical trials}

- Recent clinical trials support the efficacy and safety of radiotherapy and immunotherapy combination treatment. Future perspective

- Questions relating to the ideal dose/fractionation schemes for radiotherapy and the optimal timing of checkpoint inhibitor administration need to be addressed in future clinical studies with the aim of developing evidence-based guidelines for radiotherapy and immunotherapy.

\section{References}

Papers of special note have been highlighted as: $\bullet \bullet$ of considerable interest

1. Mole RH. Whole body irradiation; radiobiology or medicine? Br. J. Radiol. 26(305), 234-241 (1953).

-• First paper to describe the effect and to use the term 'abscopal'.

2. Abuodeh Y, Venkat P, Kim S. Systematic review of case reports on the abscopal effect. Curr. Probl. Cancer 40(1), 25-37 (2016).

3. Rees GJ, Ross CM. Abscopal regression following radiotherapy for adenocarcinoma. Br. J. Radiol. 56(661), 63-66 (1983).

-. First case report of a patient presenting with lung cancer and demonstrating an abscopal effect with radiotherapy.

4. Siva S, Callahan J, Macmanus MP, Martin O, Hicks RJ, Ball DL. Abscopal [corrected] effects after conventional and stereotactic lung irradiation of non-small-cell lung cancer. J. Thorac. Oncol. 8(8), e71-e72 (2013).

5. Gardiner RE, Jahangeer S, Forde $\mathrm{P}$ et al. Low immunogenicity in non-small-cell lung cancer; do new developments and novel treatments have a role? Cancer Metastasis Rev. 34(1), 129-144 (2015). 
6. Demaria S, Kawashima N, Yang AM et al. Immune-mediated inhibition of metastases after treatment with local radiation and CTLA-4 blockade in a mouse model of breast cancer. Clin. Cancer Res. 11(2 Pt 1), 728-734 (2005).

7. Golden EB, Demaria S, Schiff PB, Chachoua A, Formenti SC. An abscopal response to radiation and ipilimumab in a patient with metastatic non-small-cell lung cancer. Cancer Immunol. Res. 1(6), 365-372 (2013).

8. Chicas-Sett R, Morales-Orue I, Castilla-Martinez J et al. Stereotactic ablative radiotherapy combined with immune checkpoint inhibitors reboots the immune response assisted by immunotherapy in metastatic lung cancer: a systematic review. Int. J. Mol. Sci. 20(9), E2173 (2019).

9. Planchard D, Popat S, Kerr K et al. Metastatic non-small-cell lung cancer: ESMO Clinical Practice Guidelines for diagnosis, treatment and follow-up. Ann. Oncol. 29(Suppl. 4), iv192-iv237 (2018).

10. Anagnostou VK, Brahmer JR. Cancer immunotherapy: a future paradigm shift in the treatment of non-small-cell lung cancer. Clin. Cancer Res. 21(5), 976-984 (2015).

11. Brahmer JR, Govindan R, Anders RA et al. The Society for Immunotherapy of Cancer consensus statement on immunotherapy for the treatment of non-small-cell lung cancer (NSCLC). J. Immunother. Cancer 6(1), 75 (2018).

12. Postmus PE, Kerr KM, Oudkerk M et al. Early and locally advanced non-small-cell lung cancer (NSCLC): ESMO Clinical Practice Guidelines for diagnosis, treatment and follow-up. Ann. Oncol. 28(Suppl. 4), iv1-iv21 (2017).

13. Lindberg K, Nyman J, Riesenfeld Kallskog V et al. Long-term results of a prospective Phase II trial of medically inoperable stage I NSCLC treated with SBRT - the Nordic experience. Acta Oncol. 54(8), 1096-1104 (2015).

14. Glatzer M, Elicin O, Ramella S, Nestle U, Putora PM. Radio(chemo)therapy in locally advanced nonsmall cell lung cancer. Eur. Respir. Rev. 25(139), 65-70 (2016).

15. Antonia SJ, Villegas A, Daniel D et al. Overall survival with durvalumab after chemoradiotherapy in stage III NSCLC. N. Engl. J. Med. 379(24), 2342-2350 (2018).

16. Putora PM, Früh M, Kern L. The place of radiotherapy in the palliative management of NSCLC. Breathe 8(2), 134-143 (2011).

17. Novello S, Barlesi F, Califano R et al. Metastatic non-small-cell lung cancer: ESMO Clinical Practice Guidelines for diagnosis, treatment and follow-up. Ann. Oncol. 27(Suppl. 5), v1-v27 (2016).

18. Palma DA, Olson R, Harrow $S$ et al. Stereotactic ablative radiotherapy versus standard of care palliative treatment in patients with oligometastatic cancers (SABR-COMET): a randomised, Phase II, open-label trial. Lancet 393(10185), 2051-2058 (2019).

19. Gomez DR, Tang C, Zhang J et al. Local consolidative therapy vs. maintenance therapy or observation for patients with oligometastatic non-small-cell lung cancer: long-term results of a multi-institutional, Phase II, randomized study. J. Clin. Oncol. 37(18), 1558-1565 (2019).

20. Lim W, Ridge CA, Nicholson AG, Mirsadraee S. The 8(th) lung cancer TNM classification and clinical staging system: review of the changes and clinical implications. Quant. Imaging Med. Surg. 8(7), 709-718 (2018).

21. Hamilton AJ, Seid J, Verdecchia K, Chuba P. Abscopal effect after radiosurgery for solitary brain metastasis from non-small-cell lung cancer. Cureus 10(12), e3777 (2018).

22. Kuroda A, Tabuchi T, Iwami E et al. Abscopal effect of radiation on multiple lung metastases of lung adenocarcinoma: a case report. BMC Cancer 19(1), 336 (2019).

23. Cong Y, Shen G, Wu S, Hao R. Abscopal regression following SABR for non-small-cell-lung cancer: a case report. Cancer Biol. Ther. 18(1), 1-3 (2017).

24. Komatsu T, Nakamura K, Kawase A. Abscopal effect of nivolumab in a patient with primary lung cancer. J. Thorac. Oncol. 12(9), e143-e144 (2017).

25. Bitran J. The abscopal effect exists in non-small-cell lung cancer: a case report and review of the literature. Cureus 11(2), e4118 (2019).

26. Britschgi C, Riesterer O, Burger IA, Guckenberger M, Curioni-Fontecedro A. Report of an abscopal effect induced by stereotactic body radiotherapy and nivolumab in a patient with metastatic non-small-cell lung cancer. Radiat. Oncol. 13(1), 102 (2018).

27. Yaguchi D, Ichikawa M, Ito M, Okamoto S, Kimura H, Watanabe K. Dramatic response to nivolumab after local radiotherapy in pulmonary pleomorphic carcinoma with rapid progressive post-surgical recurrence. Thorac. Cancer 10(5), 1263-1266 (2019).

28. Lin X, Lu T, Xie Z et al. Extracranial abscopal effect induced by combining immunotherapy with brain radiotherapy in a patient with lung adenocarcinoma: a case report and literature review. Thorac. Cancer 10(5), 1272-1275 (2019).

29. Steel GG, Mcmillan TJ, Peacock JH. The 5Rs of radiobiology. Int. J. Radiat. Biol. 56(6), 1045-1048 (1989).

30. Ward JF. The complexity of DNA damage: relevance to biological consequences. Int. J. Radiat. Biol. 66(5), 427-432 (1994).

31. Blomgren H, Glas U, Melen B, Wasserman J. Blood lymphocytes after radiation therapy of mammary carcinoma. Acta Radiol. Ther. Phys. Biol. 13(3), 185-200 (1974).

32. Campian JL, Ye X, Brock M, Grossman SA. Treatment-related lymphopenia in patients with stage III non-small-cell lung cancer. Cancer Invest. 31(3), 183-188 (2013). 
33. Yovino S, Kleinberg L, Grossman SA, Narayanan M, Ford E. The etiology of treatment-related lymphopenia in patients with malignant gliomas: modeling radiation dose to circulating lymphocytes explains clinical observations and suggests methods of modifying the impact of radiation on immune cells. Cancer Invest. 31(2), 140-144 (2013).

34. Kalbasi A, Komar C, Tooker GM et al. Tumor-derived CCL2 mediates resistance to radiotherapy in pancreatic ductal adenocarcinoma. Clin. Cancer Res. 23(1), 137-148 (2017).

35. Seifert L, Werba G, Tiwari S et al. Radiation therapy induces macrophages to suppress T-cell responses against pancreatic tumors in mice. Gastroenterology 150(7), 1659-1672 (2016).

36. Deng L, Liang H, Burnette B et al. Irradiation and anti-PD-L1 treatment synergistically promote antitumor immunity in mice. J. Clin. Invest. 124(2), 687-695 (2014).

37. Formenti SC, Demaria S. Systemic effects of local radiotherapy. Lancet Oncol. 10(7), 718-726 (2009).

-. Stating the rationale for improvements in immune-mediated antitumor efficacy when using combined radiotherapy and immunotherapy. This review covers some of the preclinical and clinical evidence.

38. Demaria S, Ng B, Devitt ML et al. Ionizing radiation inhibition of distant untreated tumors (abscopal effect) is immune mediated. Int. J. Radiat. Oncol. Biol. Phys. 58(3), 862-870 (2004).

-• First article identifying an immune-mediated mechanism as the basis of the abscopal effect.

39. Grass GD, Krishna N, Kim S. The immune mechanisms of abscopal effect in radiation therapy. Curr. Probl. Cancer 40(1), 10-24 (2016).

40. Ng J, Dai T. Radiation therapy and the abscopal effect: a concept comes of age. Ann. Transl. Med. 4(6), 118 (2016).

41. Gardai SJ, Mcphillips KA, Frasch SC et al. Cell-surface calreticulin initiates clearance of viable or apoptotic cells through trans-activation of LRP on the phagocyte. Cell 123(2), 321-334 (2005).

42. Multhoff G, Pockley AG, Streffer C, Gaipl US. Dual role of heat shock proteins (HSPs) in anti-tumor immunity. Curr. Mol. Med. 12(9), 1174-1182 (2012).

43. Dressel R. Collaboration of heat shock protein 70 and stress-induced NKG2D ligands in the activation of NK cells against tumors. Curr. Immunol. Rev. 13(1), 56-63 (2017).

44. Apetoh L, Ghiringhelli F, Tesniere A et al. The interaction between HMGB1 and TLR4 dictates the outcome of anticancer chemotherapy and radiotherapy. Immunol. Rev. 220(1) 47-59 (2007).

45. Apetoh L, Ghiringhelli F, Tesniere A et al. Toll-like receptor 4-dependent contribution of the immune system to anticancer chemotherapy and radiotherapy. Nat. Med. 13(9), 1050-1059 (2007).

46. Andersson U, Wang H, Palmblad K et al. High mobility group 1 protein (HMG-1) stimulates proinflammatory cytokine synthesis in human monocytes. J. Exp. Med. 192(4), 565-570 (2000).

47. Ghiringhelli F, Apetoh L, Tesniere A et al. Activation of the NLRP3 inflammasome in dendritic cells induces IL-1beta-dependent adaptive immunity against tumors. Nat. Med. 15(10), 1170-1178 (2009).

48. Chakraborty M, Abrams SI, Camphausen $\mathrm{K}$ et al. Irradiation of tumor cells up-regulates Fas and enhances CTL lytic activity and CTL adoptive immunotherapy. J. Immunol. 170(12), 6338-6347 (2003).

49. Garnett CT, Palena C, Chakraborty M, Tsang KY, Schlom J, Hodge JW. Sublethal irradiation of human tumor cells modulates phenotype resulting in enhanced killing by cytotoxic T lymphocytes. Cancer Res. 64(21), 7985-7994 (2004).

50. Hallahan D, Kuchibhotla J, Wyble C. Cell adhesion molecules mediate radiation-induced leukocyte adhesion to the vascular endothelium. Cancer Res. 56(22), 5150-5155 (1996).

51. Lugade AA, Sorensen EW, Gerber SA, Moran JP, Frelinger JG, Lord EM. Radiation-induced IFN-gamma production within the tumor microenvironment influences antitumor immunity. J. Immunol. 180(5), 3132-3139 (2008).

52. Matsumura S, Wang B, Kawashima $\mathrm{N}$ et al. Radiation-induced CXCL16 release by breast cancer cells attracts effector T cells. J. Immunol. 181(5), 3099-3107 (2008).

53. Reits EA, Hodge JW, Herberts CA et al. Radiation modulates the peptide repertoire, enhances MHC class I expression, and induces successful antitumor immunotherapy. J. Exp. Med. 203(5), 1259-1271 (2006).

54. Ruocco MG, Pilones KA, Kawashima $\mathrm{N}$ et al. Suppressing $\mathrm{T}$ cell motility induced by anti-CTLA-4 monotherapy improves antitumor effects. J. Clin. Invest. 122(10), 3718-3730 (2012).

55. Son $\mathrm{CH}$, Keum JH, Yang K et al. Synergistic enhancement of NK cell-mediated cytotoxicity by combination of histone deacetylase inhibitor and ionizing radiation. Radiat. Oncol. 9 , 49 (2014).

56. Isernhagen A, Malzahn D, Viktorova $\mathrm{E}$ et al. The MICA-129 dimorphism affects NKG2D signaling and outcome of hematopoietic stem cell transplantation. EMBO Mol. Med. 7(11), 1480-1502 (2015).

57. Ganss R, Ryschich E, Klar E, Arnold B, Hammerling GJ. Combination of T-cell therapy and trigger of inflammation induces remodeling of the vasculature and tumor eradication. Cancer Res. 62(5), 1462-1470 (2002).

58. Klug F, Prakash H, Huber PE et al. Low-dose irradiation programs macrophage differentiation to an iNOS(+)/M1 phenotype that orchestrates effective T cell immunotherapy. Cancer Cell 24(5), 589-602 (2013). 
59. Spranger S, Gajewski TF. Mechanisms of tumor cell-intrinsic immune evasion. Ann. Rev. Cancer Biol. 2(1), 213-228 (2018).

60. Deng L, Liang H, Xu M et al. STING-dependent cytosolic DNA sensing promotes radiation-induced type I interferon-dependent antitumor immunity in immunogenic tumors. Immunity 41(5), 843-852 (2014).

61. Harding SM, Benci JL, Irianto J, Discher DE, Minn AJ, Greenberg RA. Mitotic progression following DNA damage enables pattern recognition within micronuclei. Nature 548(7668), 466-470 (2017).

62. Mackenzie KJ, Carroll P, Martin CA et al. cGAS surveillance of micronuclei links genome instability to innate immunity. Nature 548(7668), 461-465 (2017).

63. Vanpouille-Box C, Alard A, Aryankalayil MJ et al. DNA exonuclease Trex1 regulates radiotherapy-induced tumour immunogenicity. Nat. Commun. 8, 15618 (2017).

64. Buchbinder EI, Desai A. CTLA-4 and PD-1 pathways: similarities, differences, and implications of their inhibition. Am. J. Clin. Oncol. 39(1), 98-106 (2016).

65. Facciabene A, Motz GT, Coukos G. T-regulatory cells: key players in tumor immune escape and angiogenesis. Cancer Res. 72(9), 2162-2171 (2012).

66. Dewan MZ, Galloway AE, Kawashima N et al. Fractionated but not single-dose radiotherapy induces an immune-mediated abscopal effect when combined with anti-CTLA-4 antibody. Clin. Cancer. Res. 15(17), 5379-5388 (2009).

67. Yoshimoto Y, Suzuki Y, Mimura K et al. Radiotherapy-induced anti-tumor immunity contributes to the therapeutic efficacy of irradiation and can be augmented by CTLA-4 blockade in a mouse model. PLoS ONE 9(3), e92572 (2014).

68. Wu L, Wu MO, De La Maza L et al. Targeting the inhibitory receptor CTLA-4 on T cells increased abscopal effects in murine mesothelioma model. Oncotarget 6(14), 12468-12480 (2015).

69. Young KH, Baird JR, Savage T et al. Optimizing timing of immunotherapy improves control of tumors by hypofractionated radiation therapy. PLoS ONE 11(6), e0157164 (2016).

70. Dovedi SJ, Illidge TM. The antitumor immune response generated by fractionated radiation therapy may be limited by tumor cell adaptive resistance and can be circumvented by PD-L1 blockade. Oncoimmunology 4(7), e1016709 (2015).

-• Demonstrates the efficacy of fractionated radiotherapy and antiprogrammed death ligand 1 inhibitor combination.

71. Dovedi SJ, Adlard AL, Lipowska-Bhalla G et al. Acquired resistance to fractionated radiotherapy can be overcome by concurrent PD-L1 blockade. Cancer Res. 74(19), 5458-5468 (2014).

72. Hettich M, Lahoti J, Prasad S, Niedermann G. Checkpoint antibodies but not T cell-recruiting diabodies effectively synergize with TIL-inducing gamma-irradiation. Cancer Res. 76(16), 4673-4683 (2016).

73. Twyman-Saint Victor C, Rech AJ, Maity A et al. Radiation and dual checkpoint blockade activate non-redundant immune mechanisms in cancer. Nature 520(7547), 373-377 (2015).

-• Preclinical evidence of the optimal timing for associating radiotherapy with checkpoint inhibitors.

74. Postow MA, Callahan MK, Barker CA et al. Immunologic correlates of the abscopal effect in a patient with melanoma. N. Engl. J. Med. 366(10), 925-931 (2012)

75. Hiniker SM, Chen DS, Reddy S et al. A systemic complete response of metastatic melanoma to local radiation and immunotherapy. Transl. Oncol. 5(6), 404-407 (2012).

76. Hiniker SM, Reddy SA, Maecker HT et al. A prospective clinical trial combining radiation therapy with systemic immunotherapy in metastatic melanoma. Int. J. Radiat. Oncol. Biol. Phys. 96(3), 578-588 (2016).

77. Takeshima T, Chamoto K, Wakita D et al. Local radiation therapy inhibits tumor growth through the generation of tumor-specific CTL: its potentiation by combination with Th1 cell therapy. Cancer Res. 70(7), 2697-2706 (2010).

78. Marciscano AE, Ghasemzadeh A, Nirschl TR et al. Elective nodal irradiation attenuates the combinatorial efficacy of stereotactic radiation therapy and immunotherapy. Clin. Cancer Res. 24(20), 5058-5071 (2018).

79. Nestle U, De Ruysscher D, Ricardi U et al. ESTRO ACROP guidelines for target volume definition in the treatment of locally advanced non-small-cell lung cancer. Radiother. Oncol. 127(1), 1-5 (2018).

80. Glatzer M, Rittmeyer A, Muller J et al. Treatment of limited disease small cell lung cancer: the multidisciplinary team. Eur. Respir. J. 50(2), (2017).

81. Schaue D, Ratikan JA, Iwamoto KS, Mcbride WH. Maximizing tumor immunity with fractionated radiation. Int. J. Radiat. Oncol. Biol. Phys. 83(4), 1306-1310 (2012).

82. Frey B, Ruckert M, Weber $\mathrm{J}$ et al. Hypofractionated irradiation has immune stimulatory potential and induces a timely restricted infiltration of immune cells in colon cancer tumors. Front. Immunol. 8, 231 (2017).

83. Siva S, Macmanus MP, Martin RF, Martin OA. Abscopal effects of radiation therapy: a clinical review for the radiobiologist. Cancer Lett. 356(1), 82-90 (2015).

84. Finkelstein SE, Timmerman R, Mcbride WH et al. The confluence of stereotactic ablative radiotherapy and tumor immunology. Clin. Dev. Immunol. 2011, 439752 (2011). 
85. Buchwald ZS, Wynne J, Nasti TH et al. Radiation, immune checkpoint blockade and the abscopal effect: a critical review on timing, dose and fractionation. Front. Oncol. 8, 612 (2018).

86. Manapov F, Roengvoraphoj O, Dantes M, Marschner S, Li M, Eze C. Pneumonitis in irradiated lungs after nivolumab: a brief communication and review of the literature. J. Immunother. 41(2), 96-99 (2018).

87. Louvel G, Bahleda R, Ammari S et al. Immunotherapy and pulmonary toxicities: can concomitant immune-checkpoint inhibitors with radiotherapy increase the risk of radiation pneumonitis? Eur. Respir. J. 51(1), 1701737 (2018).

88. Johnson DB, Balko JM, Compton ML et al. Fulminant myocarditis with combination immune checkpoint blockade. N. Engl. J. Med. 375(18), 1749-1755 (2016).

89. Ko EC, Raben D, Formenti SC. The integration of radiotherapy with immunotherapy for the treatment of non-small-cell lung cancer. Clin. Cancer Res. 24(23), 5792-5806 (2018).

-• Reports the safety of combined radiotherapy and immunotherapy treatment.

90. Formenti SC, Rudqvist NP, Golden E et al. Radiotherapy induces responses of lung cancer to CTLA-4 blockade. Nat. Med. 24(12), 1845-1851 (2018).

91. Shaverdian N, Lisberg AE, Bornazyan K et al. Previous radiotherapy and the clinical activity and toxicity of pembrolizumab in the treatment of non-small-cell lung cancer: a secondary analysis of the KEYNOTE-001 Phase I trial. Lancet Oncol. 18(7), 895-903 (2017).

92. Garon EB, Rizvi NA, Hui R et al. Pembrolizumab for the treatment of non-small-cell lung cancer. N. Engl. J. Med. 372(21), 2018-2028 (2015).

-• Describes the efficacy of pembrolizumab in combination with extracranial radiotherapy with regard to progression-free and overall survival.

93. Gettinger SN, Horn L, Gandhi L et al. Overall survival and long-term safety of nivolumab (anti-programmed death 1 antibody, BMS-936558, ONO-4538) in patients with previously treated advanced non-small-cell lung cancer. J. Clin. Oncol. 33(18), 2004-2012 (2015).

94. Theelen W, Peulen H, Lalezari F et al. Randomized Phase II study of pembrolizumab after stereotactic body radiotherapy (SBRT) versus pembrolizumab alone in patients with advanced non-small-cell lung cancer: the PEMBRO-RT study. J. Clin. Oncol. 36(Suppl. 15), 9023-9023 (2018).

95. Antonia SJ, Villegas A, Daniel D et al. Durvalumab after chemoradiotherapy in stage III non-small-cell lung cancer. N. Engl. J. Med. 377(20), 1919-1929 (2017).

-• Reports the efficacy of durvalumab in patients with non-small-cell lung cancer that had not progressed after platinum-based chemo-radiotherapy. 